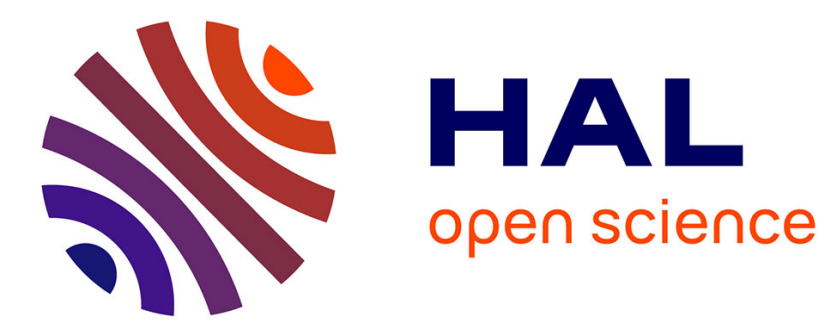

\title{
Note sur l'emploi et l'origine de la notion de représentation en sciences cognitives
}

Jean Lassègue

\section{To cite this version:}

Jean Lassègue. Note sur l'emploi et l'origine de la notion de représentation en sciences cognitives. Intellectica - La revue de l'Association pour la Recherche sur les sciences de la Cognition (ARCo), 1993, 1993/2 (17), pp.199-212. halshs-00008858

\section{HAL Id: halshs-00008858 \\ https://shs.hal.science/halshs-00008858}

Submitted on 6 Feb 2006

HAL is a multi-disciplinary open access archive for the deposit and dissemination of scientific research documents, whether they are published or not. The documents may come from teaching and research institutions in France or abroad, or from public or private research centers.
L'archive ouverte pluridisciplinaire HAL, est destinée au dépôt et à la diffusion de documents scientifiques de niveau recherche, publiés ou non, émanant des établissements d'enseignement et de recherche français ou étrangers, des laboratoires publics ou privés. 


\author{
Note \\ sur l'emploi et l'origine \\ de la notion de représentation \\ en sciences cognitives
}

\title{
Résumé :
}

Les sciences cognitives ont hérité du questionnement philosophique sur la nature de la représentation en considérant la notion de représentation symbolique du réel comme fondamentale pour la description du fonctionnement de l'esprit. Mais la représentation n'est pas seulement un contenu mental qui serait susceptible d'être manipulé formellement. On retrouve la notion de représentation à trois niveaux distincts : un niveau intuitif, un niveau formel et discret, un niveau physique et continu. Seul le niveau formel et discret est pris en compte quand on parle de fonctionnement représentationnel de l'esprit. Réintroduire une réflexion plus générale sur la notion de représentation permet de retrouver l'origine cartésienne de la notion et de mettre au jour son aspect herméneutique.

\section{Abstract : Remarks on the use and origin of the notion of representation in the cognitive sciences}

Cognitive sciences have inherited from traditional philosophy the question of the nature of representation, because they consider the notion of symbolic representation of reality as a fundamental aspect of how the mind works. But representations are not only mental contents that one can manipulate formally. The notion is in fact present at three different levels : an intuitive level, a formal and discrete level and a continuous and physical one. Only the formal and discrete level is taken into account when one speaks of a representational way of how the mind works. If one reintroduces a more general conception of representation, one can trace the notion back to its cartesian origin and show its hermeneutical apsects. 


\section{Introduction}

L'enquête que nous allons mener porte sur la notion de représentation ${ }^{1}$, dont les sciences cognitives ont hérité après une longue histoire philosophique, qu'il faut faire remonter à l'époque de Descartes, c'est-à-dire à l'époque de la naissance d'une physique mathématique. En replaçant la notion dans une perspective historique, on se rend compte qu'elle n'est pas une notion qui serait donnée comme entièrement constituée sous la forme d'un objet abstrait, mais qu'elle est au contraire le résultat d'un processus ininterrompu d'interprétation théorique. Dès lors, la notion de représentation n'a pas de statut scientifique définitif et n'est peut-être pas destinée à en avoir un, mais elle possède en revanche un rôle herméneutique indispensable pour mener une enquête sur les buts que se propose d'atteindre une "science de l'esprit".

En postulant l'existence d'un niveau cognitif spécifique au mental, conçu comme pleinement autonome par rapport aux processus neuronaux, les sciences cognitives ont accordé une place capitale à la notion de représentation symbolique du réel $^{2}$. Elles considèrent en particulier que le domaine de la représentation peut être adéquatement décrit grâce à une méthodologie mise au point à partir de formalismes dérivés des mathématiques et de la logique : la représentation symbolique est en effet considérée comme susceptible d'un traitement de nature calculatoire. A partir de là, et par une sorte de radicalisation, le domaine de la représentation apparaît tout entier assimilable à une machinerie logique et l'ordinateur digital apparaît naturellement comme le modèle le plus adéquat pour rendre compte du fonctionnement de ce domaine, dont on postule qu'il sert de soubassement à la vie de la pensée.

Sans faire la généalogie des rapports multiformes de la logique et des mathématiques depuis la constitution d'une logique mathématique proprement dite, on peut isoler un certain nombre de résultats qui ont contribué à rendre possible la construction des premiers ordinateurs ${ }^{3}$ et à en faire, pour les sciences cognitives, des modèles explicatifs pour le fonctionnement du domaine de la représentation. Ces résultats tournent autour de ce qu'il est convenu d'appeler la "thèse de Church".

\section{La thèse de Church et les thèses de Turing}

\section{La thèse de Church}

1 Comme le choix du mot "note" l'indique, il ne s'agit pas de décrire toutes les facettes de la notion de représentation mais seulement d'en éclairer un aspect fondamental.

2 Cf par exemple, Pylyshyn Z. (1984), Introduction.

3 Cf Davis M. (1987) et (1988). 
Dans le cadre de la logique des prédicats du premier ordre, l'Entscheidungsproblem pose le problème de savoir si on peut toujours trouver un algorithme général qui décide en temps fini de la déductibilité ou de la nondéductibilité d'un énoncé quelconque : y-a-t-il un algorithme qui puisse décider si, pour tout problème mathématique, il existe un algorithme qui résoudrait le problème envisagé ? Church montre qu'il est possible de passer en revue la classe de tous les algorithmes possibles et qu'il n'y a pas de réponse affirmative à cette question ${ }^{4}$.

Le raisonnement de Church semble exiger que l'on précise la notion d'algorithme par des moyens algorithmiques. En effet, la difficulté provient de ce qu'il faudrait que la notion d'opération effectuée par l'esprit puisse s'extérioriser entièrement dans la notion formelle. De quels moyens dispose-t-on pour s'en assurer ? Les procédures peuvent-elles elles-mêmes être qualifiées d'algorithmes ? On voit que s'ouvre ici une régression indéfinie. Pour sortir de cette difficulté, il faut réussir à exhiber une notion équivalente à la notion d'algorithme mais qui ne conduise pas comme elle à produire, psychologiquement, une régression indéfinie. Il faut, pour ce faire, rompre avec le caractère informel de la notion d'algorithme en exhibant une nouvelle notion qui soit formelle et qui couvre tout le champ couvert par la notion intuitive. Il devient alors possible d'opérer une identification entre la notion d'algorithme et cette notion équivalente. C'est l'objet de la thèse de Church, qui montre l'identité entre plusieurs notions équivalentes entre elles, tout en rapportant ces notions à la notion informelle d'algorithme. La thèse opère cette identification, dont le fondement n'est pas formel mais seulement psychologique : la référence au psychologique s'est ainsi déplacée d'un terme (la notion informelle d'algorithme) à un rapport (celui que l'on constitue entre la notion informelle d'algorithme et les notions formelles équivalentes). La thèse de Church identifie en effet la notion de fonction effectivement calculable d'entiers positifs à la notion de fonction récursive d'entiers positifs ou à la notion de fonction $\lambda$-définissable d'entiers positifs, tout en faisant remarquer que les justifications de cette identification ne peuvent pas être adéquates parce que la notion d'effectivité (ou d'algorithme) est intuitive tandis que les notions de récursivité ou de $\lambda$-définissabilité sont formelles 5 :

«On considère que cette définition est justifiée par les considérations qui suivent, si tant est qu'une justification positive puisse jamais être obtenue quant au choix d'une définition formelle qui corresponde à une notion intuitive. »

\footnotetext{
4 Church A. (1936a).

5 Church A. (1936b).
} 
La thèse de Church vise à définir l'essence du calcul. C'est en particulier à partir d'elle qu'il est possible de donner tout son sens à l'intertraductibilité 6 des langages formels, du $\lambda$-calcul de Church aux fonctions récursives de Herbrand-Gödel et aux langages de programmation d'aujourd'hui. De ce point de vue, la thèse de Church permet de définir le calcul en déterminant les conditions linguistiques formelles auxquelles il doit être soumis pour être considéré comme calcul.

Notons que Post réagit à l'énoncé de la thèse de Church en faisant remarquer qu'exprimer cette thèse sous la forme d'une définition revient à occulter son aspect informel, aspect qui manifeste en fait l'existence d'une limitation interne aux pouvoirs mathématiques des êtres humains, dans la mesure où cette thèse exige d'être continuellement reconfirmée ${ }^{7}$ :

« Mais masquer l'identification derrière une définition occulte le fait qu'une découverte fondamentale concernant les limitations $\mathrm{du}$ pouvoir mathématique de Homo Sapiens a été réalisée et nous rend aveugle au besoin de sa continuelle vérification. »

C'est précisément l'un des aspects du travail de Turing dans [Turing 19361937], que de s'interroger directement sur les pouvoirs mathématiques des êtres humains, c'est-à-dire de tenter de mener une investigation mathématico-psychologique, sans aucun équivalent jusqu'alors 8 . Aussi peut-on dire qu'il y a bien une "thèse de Turing" de nature psychologique, qu'il ne faut pas assimiler à la thèse de Church, de nature linguistique et définitionnelle : même si la thèse de Turing est formellement identique à la thèse de Church, elle ne l'est pas psychologiquement, dans la mesure où Turing tente d'apporter des arguments informels spécifiques à l'identification opérée dans la thèse de Church entre l'effectif et le récursif ${ }^{9}$. Ces arguments consistent à introduire le concept, désormais classique, de machine de Turing.

\section{La thèse de Turing $n^{\circ} 1$}

La thèse de Turing porte sur la nature psychologique de celui qui calcule, que Turing appelle le "calculateur" [“computer"]10 : elle entre dans une réflexion scientifique sur l'essence de l'esprit. Elle consiste à identifier les étapes franchies par

\footnotetext{
6 Par exemple, pour montrer que les fonctions primitivement récursives sont $\lambda$-définissables, on vérifie qu'il est possible de traduire au moyen d'un terme du $\lambda$-calcul chaque procédé d'engendrement servant à définir la classe de ces fonctions. Cf. Barendregt Henk P. (1977), pp. 1103-1104.

7 Post E. (1936).

8 Turing A. M. (1936-1937).

9 Church apporte aussi des arguments à cette thèse. On les trouve résumés dans Gandy R., (1988).

10 Turing A. M. (1936-1937).
} 
l'être humain en train de calculer et les états d'une machine abstraite effectuant une liste finie d'instructions aboutissant à un état terminal, interprétable comme le résultat d'un calcul ${ }^{11}$ :

« Nous pouvons comparer un homme en train de calculer un nombre réel à une machine qui n'est capable que d'un nombre fini de conditions $\mathrm{q}_{1}, \mathrm{q}_{2}, \ldots, \mathrm{q}_{R}$, et qui seront appelées "m-configurations". »

Turing décrit les étapes que l'être humain doit accomplir pour mener le calcul à bien et montre que chaque étape prise séparément peut être effectuée par une machine. On peut en particulier démontrer l'existence d'une certaine classe de machines, les "machines universelles", capables d'intégrer à leur propre liste d'instructions la liste d'instructions d'une autre machine : la description de la première machine joue alors le rôle d'un argument de fonction pour la seconde. C'est cette imitation fonctionnelle qui rend possible l'universalité de cette classe de machines, dans la mesure où toutes fonctions d'entiers à valeurs entières sont calculables par une machine de cette classe.

Deux principes sont à l'œuvre pour justifier l'équivalence entre un calcul effectué par un être humain et le même calcul effectué par une machine ${ }^{12}$ : un principe de détermination, de nature déterministe (les étapes du calcul sont réglées selon un ordre immuable) et un principe de finitude, de nature psychologique (l'esprit humain ne peut percevoir qu'un nombre fini de choses à un instant donné). Le caractère essentiellement discret de la façon dont le calcul se manifeste à l'esprit en découle. L'identification, psychologiquement et non formellement fondée, entre la procédure suivie par l'être humain qui calcule et celle que suit la machine manifeste le discret comme représentant l'essence mécanique du calcul. J'appellerai cette identification "Thèse de Turing $n^{\circ} 1$ ".

\section{La thèse de Turing $n^{\circ} 2$}

Il y a en effet une seconde thèse défendue par Turing dans un article ultérieur, "Computing Machinery and Intelligence"13 et que nous appelons ici "Thèse de Turing $\mathrm{n}^{\circ} 2^{\prime}$, bien qu'elle ne soit pas répertoriée dans la littérature sous un sigle quelconque. Elle porte cette fois non sur l'identification abstraite entre un être humain et une machine mais sur leur identification physique réciproque. Cette thèse a trait, pourrait-on dire, à la physique de l'esprit et entre donc, elle aussi, dans une réflexion scientifique sur sa nature. Elle s'énonce ainsi : pour réussir à faire la différence entre un être humain

\footnotetext{
11 Turing A. M. (1936-1937).

12 Cf. Hao Wang (1974), p. 92.

13 Turing A. M.,(1950).
} 
et un ordinateur, il faut opérer sur eux une comparaison d'un point de vue physique. Par exemple, il faut être capable de distinguer le fait que l'ordinateur n'est pas constitué de tissus vivants, comme l'être humain. Cette différenciation s'opère sur le fond d'une identité commune qui consiste à reconnaître que les machines et les êtres humains sont, en tant qu'entités physiques, de nature continue, bien qu'on puisse toujours les considérer du point de vue du discret 14 :

« Les ordinateurs digitaux pris en considération dans la dernière section peuvent être rangés dans la classe des "machines à états discrets". Ce sont des machines qui se meuvent par bonds soudains ou qui sautent d'un état bien défini à un autre. Ces états sont suffisamment distincts pour que soit éliminée la possibilité d'une confusion entre eux. Au sens strict, il n'existe pas de machines semblables. Tout se meut continûment. Mais il y a beaucoup d'espèces de machines que l'on peut considérer comme des machines à états discrets. »

Quelles machines parvient-on à considérer comme discrètes ? En fait, tout calcul, en tant que calcul, peut être effectué discrètement par une machine, comme l'a prouvé Turing dans “On Computable Numbers”. Or de même que l'être humain est assimilable à une machine discrète quand il effectue abstraitement un calcul, selon la thèse de Turing $\mathrm{n}^{\circ} 1$, de même on peut le ranger, avec les machines, dans la catégorie de ce qui est physiquement continu. Turing retrouve sans doute ici cette mathématique du continu que von Neumann, à la même époque, appelait également de ses vœux dans l'étude des réseaux de neurones, parce que les neurones réels ne lui semblaient pas soumis à un mode de fonctionnement discret ${ }^{15}$. Remarquons que le concept de continu, présent dans l'esprit pour comparer être humain et ordinateur du point de vue physique, dépasse le cadre discret de la thèse que Turing soutient concernant la nature discrète de l'esprit. Turing se donne donc la possibilité de penser la nature physique par-delà le registre discret du calculable quand il attribue à la nature physique le caractère de continuité.

Les deux thèses de Turing forment ainsi une analogie : de même que l'être humain est assimilable à une machine discrète du point de vue intellectuel propre au calcul, de même on peut ranger les machines et l'être humain sous la notion physique de continu quand on se place du point de vue de leur fonctionnement réel. C'est cette analogie qui fonde, chez Turing, la possibilité d'une science de l'esprit.

\footnotetext{
14 Turing A. M., (1950), p. 439.

15 Cf. Von Neumann J. (1951), pp. 19-31 et (1958), p. 44. Cf, pour ce débat, Mosconi J. (1989), pp. 209 sq.
} 
Remarquons que les trois thèses que nous venons d'exposer, la thèse de Church et les thèses de Turing $n^{\circ} 1$ et $n^{\circ} 2$, délimitent trois registres, le premier intuitif et psychologique (puisqu'on vise la réduction des opérations mentales à un calcul), le second algorithmique et formel (qui accueille la forme générale de toute opération calculable) et le troisième continu et physique, dont nous venons de parler. Ces trois thèses sont nécessaires pour fonder une théorie de la représentation en sciences cognitives.

\section{La théorie de la représentation en sciences cognitives}

En généralisant la remarque de Post déjà citée, on peut dire que la thèse de Church ainsi que les thèses de Turing qui s'y rapportent, exigent, de par leur nature essentiellement psychologisante, d'être continuellement reconfirmées, c'est-à-dire d'être rapportées à la source de leur production qui est le sujet lui-même. Aussi le travail continuel de confirmation est-il une tâche infinie dévolue au sujet par lui-même, dès lors qu'il se donne pour objet de délimiter un domaine du calculable qui est lui aussi infini. Le discret qui manifeste l'essence mécanique du calcul est bien alors le nom donné au principe de production de toute représentation, principe qui trace les limites entre ce qui est légalement représentable par le sujet (à savoir le rapport qui se manifeste par le discret entre l'effectivité et le récursif et qui s'exprime dans la thèse de Church) et le non-représentable (à savoir le rapport qui se manifeste par le continu entre l'effectivité et le non-récursif et qui s'exprime dans la seconde thèse de Turing) qui est néanmoins pensable. Ce domaine du pensable en excès sur sa représentation se manifeste dans le domaine de la représentation par le biais de l'infini de la réitération au sein du discret : la tâche de confirmation ou de reprise à laquelle s'assigne le sujet manifeste, par son caractère infini, l'existence de ce qui dépasse le discret de la représentation tout en s'exprimant en elle. Ainsi voit-on apparaître, par le biais de la notion de représentation, une solidarité entre les trois registres de l'intuitif, du formel et du physique et ce, par le biais de trois concepts qui les caractérisent respectivement, l'infini, le discret et le continu. Mais cette solidarité ne s'exprime pas sous l'apparence d'un objet calculable mais bien sous celle de ce qui, au sein du calcul, le dépasse.

On voit mieux maintenant pourquoi le choix de l'ordinateur comme modèle de la pensée en sciences cognitives a des conséquences complexes sur l'interprétation que l'on donne de la notion de représentation. Telle qu'on l'a décrite, l'interprétation reçue en sciences cognitives tend à limiter la portée de la notion au registre du formel discret sans parvenir cependant à l'y cantonner. Dès lors, la question des rapports qu'entretiennent les trois registres resurgit comme une sorte de point aveugle. Il est 
cependant possible d'éclaircir la nature de cette théorie de la représentation quand on prend conscience qu'elle n'est pas née avec le paradigme de l'ordinateur. En particulier, les trois registres, intuitif, formel et physique, mis en place par la thèse de Church et celles de Turing, ne sont pas une nouveauté d'un point de vue philosophique. On les trouve réunis chez Descartes et ils ne peuvent pas ne pas être interpétés aujourd'hui comme les prémisses d'une théorie de la représentation.

\section{L'héritage cartésien de la notion de représentation}

Le terme de représentation n'est pas fréquent chez Descartes bien qu'il ait élaboré le cadre de pensée nécessaire à sa constitution, cadre qui renvoie à la fois aux domaines de la perception, de l'âme et des mathématiques ${ }^{16}$. C'est par le biais du domaine mathématique que j'aborde la théorie cartésienne, dans la mesure où c'est précisément de cet héritage mathématique dont il est ici question.

Descartes conçoit l'existence d'un rapport qui relie les trois registres intuitif, formel et physique par le biais de la notion de représentation. La représentation n'est donc pas un terme que l'on poserait au départ comme un acquis mais elle est plutôt le résultat d'un processus d'interprétation qui tente de rendre compte de la solidarité entre registres hétérogènes.

Sont considérées comme représentations les idées présentes à l'esprit dont il est possible de retracer la construction. La construction, par son style d'effectivité, occupe le registre de ce que l'on a appelé plus haut, dans le cadre de la logique mathématique, le formel. Il s'agit d'une notion centrale de la géométrie analytique : elle permet la mise en correspondance entre les points de l'espace euclidien et les solutions d'une équation algébrique. Une courbe est considérée comme construite quand la correspondance entre l'algèbre et la géométrie est susceptible de s'exprimer par une mesure rationnelle, c'està-dire comme un résultat fini exprimable par des nombres entiers ou rationnels.

Mais pour Descartes, la notion de construction qu'il a inventée pour ses besoins en mathématique dépasse largement ce cadre. En particulier, on peut construire des points d'abscisse $\sqrt{2}$ sur une droite et plus généralement, accéder à des points de coordonnée irrationnelle. La généralité de cette notion ne permet pas néanmoins de saisir sa propre condition de possibilité : le domaine du mouvement physique. Le mouvement échappe à toute construction parce qu'il enveloppe une continuité, qui n'est pas susceptible d'être exprimée par une mesure euclidienne ${ }^{17}$. C'est pourquoi Descartes

16 Respectivement dans les Principes de la philosophie (Première partie), les Méditations métaphysiques (Troisième méditation) et la Géométrie (Deuxième partie).

17 Elle enveloppe une infinité non-dénombrable. Cf. Vuillemin J. (1960), p. 85. 
limite sa physique à l'étude des systèmes à l'équilibre tout en sachant, comme Turing après lui, que tout se meut continûment. Mais par ailleurs, c'est le mouvement qui permet la construction parce que c'est grâce au mouvement qu'il est possible de rendre compte du caractère génétique propre à toute construction, c'est-à-dire de la possibilité de passer, en géométrie, d'un point à un autre sur une courbe et en algèbre, d'un nombre à un autre dans les solutions d'une équation. Or ce mouvement à l'origine de toute construction se manifeste dans deux registres : celui de l'esprit d'une part et celui de la machine de l'autre.

Du point de vue de l'esprit, il faut, pour réussir à engendrer des constructions, que celui-ci soit capable de mettre en correspondance les figures continues et les nombres discrets : ce n'est possible que si l'esprit est en lui-même continu et discret. Pour posséder ces deux caractéristiques, l'esprit doit posséder une direction. La direction exprime en effet cette dualité : la direction est d'une part un mouvement, qui enveloppe de ce fait une virtualité non-mesurable parce qu'il peut être indéfiniment prolongé mais d'autre part, la direction possède un principe interne de construction selon un ordre, principe qui permet de mettre en rapport les points de l'espace géométrique dans des figures et les suites de nombres dans les solutions des équations algébriques. Il y a donc une partie de ce qui relève de l'esprit qui reste inaccessible à toute construction mathématique, le mouvement continu de l'esprit, bien que cette partie se manifeste de façon géométrique dans l'idée de direction de l'esprit.

Du point de vue de la machine, Descartes utilise, dans la Géométrie, une machine de son invention qui permet de tracer des courbes. La machine de Descartes est une sorte de compas que l'on aurait placé à l'horizontale et dans lequel on aurait fixé à intervalles réguliers des équerres mobiles sur lesquelles sont fixées des pointes permettant de tracer des traits. On imprime aux équerres situées près de l'angle du compas un mouvement continu ${ }^{18}$ qui fait bouger les pointes des équerres : celles-ci, en se déplaçant, tracent des courbes. Le mouvement continu de la machine est à l'origine des constructions de la géométrie analytique et entre dans un rapport analogique avec le mouvement de l'esprit19: de même que dans l'esprit en effet, le mouvement continu imprimé aux équerres de la machine est la condition de possibilité de toute construction géométrique tout en échappant lui-même à la construction.

Une dernière remarque de Descartes vient compléter la théorie de la construction : la machine de la Géométrie trace plus de courbes que l'analytique

\footnotetext{
18 Descartes (1637), p. 390.

19 Il ne s'agit que d'une analogie car le mouvement continu de l'esprit doit être actuel alors que le mouvement continu nécessaire au mouvement de la machine n'est que potentiel. Cf. les remarques de Lachterman (1989), pp. 198-204.
} 
cartésienne n'est capable d'en construire 20 . Pour employer quelque peu abusivement un langage moderne, on pourrait dire qu'il y a plus d'objets traçables qu'il n'y a de types formellement constructibles qui leur correspondent. Cette remarque est véritablement paradoxale : comment accorder le statut de courbe à ce qui n'est pas construit ? C'est le propre d'une théorie de la représentation que de réussir à définir à la fois le domaine dans lequel un objet peut être représenté et le domaine qui dépasse le cadre du représentable, en l'occurrence le mouvement continu. C'est précisément ce qui se produit chez Descartes dans sa définition de certaines "courbes", comme par exemple celle de la courbe logarithme qui échappe à la construction au moyen de la machine à équerres mobiles : elles apparaissent, sans qu'il soit possible de les maîtriser dans une représentation qui les rendraient "recevables en géométrie" et manifestent la tâche infinie propre au sujet, qui consiste à tenter d'opérer une discrétisation nouvelle d'un domaine continu de la pensée en faisant le choix de nouvelles "primitives" pour sa construction.

Ainsi faut-il reconnaître l'existence de rapports idéels entre le registre intuitif de l'esprit capable de reconnaître des courbes qui existent sur un mode non-constructif et le registre physique qui manifeste, par l'intermédiaire du mouvement continu de la machine, un ordre qui dépasse le registre du construit. Ces rapports permettent de définir ainsi à la fois ce qui existe dans le domaine de la représentation -et qui exige d'être construit- et ce qui dépasse le domaine de la représentation -et qui, tout en méritant le nom d'idée, n'est interprétable qu'en images-. La machine dans son mouvement continu apparaît en effet à l'esprit comme l'image (et non la représentation au sens cartésien, qui demande une construction) de son propre mouvement : elle ne sert donc pas d'abord à calculer puisqu'elle ne construit pas des représentations, mais à donner une image du caractère incalculable du mouvement continu de l'esprit, par ailleurs générateur des constructions. Ce que l'utilisation de la notion de machine tend à montrer chez Descartes, c'est qu'il n'est pas possible de posséder constructivement, c'est-à-dire dans une représentation, les trois registres de l'intuitif, du formel et du physique mais, parce que la théorie philosophique de la représentation définit aussi son autre, à savoir le non-construit, il est possible, grâce à ce que la machine produit et qui est interprété comme image du mouvement de l'esprit21, d'avoir l'idée de la parenté entre ces trois registres.

\footnotetext{
20 Par exemple, Descartes reconnaît l'existence de la courbe logarithme (lettre à Mersenne du 12 Septembre 1638, p. 360), à partir du tracé de certains de ses points par la machine, mais il est incapable de justifier mathématiquement dans une construction ce qu'il est cependant capable de reconnaître intuitivement.

21 La machine ne produit pas d'images : c'est l'esprit, dans son mouvement sur lui-même, qui interprète le caractère éparpillé et sans ordre des points qu'il a construits dans l'espace comme formant cependant une courbe. C'est la construction mécanique elle-même qui produit donc, comme dans un choc en retour sur l'esprit, la
} 


\title{
Remarques finales
}

Descartes semble donc avoir le premier posé les principes idéaux -en l'occurrence les trois registres de l'intuitif, du formel et du physique- qui rendent possible à la fois une recherche sur un mode scientifique -c'est-à-dire une détermination de l'essence de l'objet-et une interprétation sur un mode philosophique -c'est-à-dire une détermination de l'essence de la représentation. Après trois siècles de révolutions mathématiques et physiques, le cadre interprétatif qu'il a constitué demeure, sans doute parce que le projet rationnel du XVIIème siècle, la mathesis universalis, est d'essence représentationnelle : il s'agit d'essayer de penser mathématiquement la nature, c'est-à-dire de maîtriser les rapports entre les objets matériels à partir de calculs abstraits mais qui renvoient à une géométrie.

On peut assurément suspendre l'enquête de nature herméneutique menée à partir de la notion de représentation et l'utiliser telle quelle, en prenant pour un fait objectivement descriptible l'existence de son domaine opératoire, c'est-à-dire en ne considérant que son caractère discret. Les représentations existeraient alors comme des propositions au sens de la logique des prédicats et on devrait les considérer comme susceptibles d'être syntaxiquement manipulées dans un univers discret. Mais on laisserait alors de côté l'idée de limite entre représentable discret et non-représentable continu, limite qui ne peut pas être appréhendée sous la forme d'un objet propositionnel $^{22}$. C'est en se fondant sur la permanence de cette idée à travers l'histoire de la mathesis universalis que l'on peut à bon droit conférer à la notion de représentation un rôle herméneutique.

\author{
Jean Lassègue
}

CNRS-CREA

$\underline{\text { Bibliographie }}$

Barendregt Henk P. (1977), “The Type-free Lambda Calculus, in [Barwise J. éd., Handbook of Mathematical Logic, North-Holland, pp. 1091-1132].

perspective sémantique qui consiste à reconnaître dans l'éparpillement des points le tracé d'une courbe noncalculable.

22 J'ai tenté de montrer dans un autre étude portant sur l'œuvre de Turing que celui-ci vivait la limitation au cadre du représentable discret sur le mode du "remords". Cf. Lassègue J., (1993). 
Church A. (1936a), “A note on the Entscheidungsproblem”, Journal of Symbolic Logic, vol. 1 n³, réédité dans [Davis M., The Undecidable, Raven Press, Hewlett, New York, 1965 pp. 108-115].

Church A. (1936b), “An unsolvable problem of Elementary Number Theory”, American Journal of Mathematics, 58, p. 356, réédité dans [Davis M., The Undecidable, Raven Press, Hewlett, New York, 1965 pp. 88-107].

Davis M. (1987), "Mathematical Logic and the Origin of Modern Computers", Studies in the Working of Mathematics, The Mathematical Association of America, réédité dans [Herken R. éd.,The Universal Turing machine, Oxford Science Publications, Oxford University Press, 1988, pp. 149-174].

Davis M. (1988), "Influences of Mathematical Logic on Computer Science” in [Herken R. éd.,The Universal Turing machine, Oxford Science Publications, Oxford University Press, 1988, pp. 315-326].

Descartes (1637), Géométrie, éd. Adam et Tannery, Euvres Complètes, t.VI, réédition [Vrin, Paris, 1982].

Descartes, lettre à Mersenne du 12 Septembre 1638, Correspondance, éd. Adam et Tannery, Euvres Complètes, t.

II, réédition [Vrin, Paris, 1982, p. 360].

Gandy R. (1988), “The Confluence of Ideas in 1936” in [Herken R. éd.,The Universal Turing machine, Oxford Science Publications, Oxford University Press, 1988, pp. 55-111].

Hao Wang (1974), From Mathematics to Philosophy, Routledge and Kegan Paul, Londres.

Lachterman D. R. (1989), The Ethics of Geometry, Routledge and Kegan Paul, New York et Londres.

Lassègue J. (1993), "Le test de Turing et l'énigme de la différence des sexes" in [Anzieu D. éd., Les Contenants de pensée, Dunod, Paris, à paraître].

Mosconi J. (1989), La constitution de la théorie des automates, thèse de doctorat d'Etat, Université de Paris I, imprimé par l'Atelier National de reproduction des thèses, Université de Lille III.

Post E. (1936), "Finite Combinatory Processes - Formulation I”, Journal of Symbolic Logic, I, réédité dans [Davis M., The Undecidable, Raven Press, Hewlett, New York, 1965, pp. 288-291].

Pylyshyn Z. (1984), Computation and Cognition, Toward a Foundation for Cognitive Science, A Bradford Book, MIT Press, Cambridge, Massachussetts.

Turing A. M. (1936-1937), 'On Computable Numbers with an Application to the Entscheidungproblem', Proceedings of the London Mathematical Society, 42, pp. 230-265, réédité dans [Davis M., The Undecidable, Raven Press, Hewlett, New York, 1965, pp. 115-154].

Turing A. M. (1950), “Computing Machinery and Intelligence”, Mind, Vol. LIX. n 236, pp. 433-460.

Von Neumann J. (1951), "Some of the problems concerning digital notions in the central nervous sytem”, édité dans [H. von Foerster éd., Cybernetics, pp. 19-31].

Von Neumann J. (1958), The Computer and the Brain, The Silliman Lectures, Yale University Press.

Vuillemin J. (1960), Mathématiques et métaphysiques chez Descartes, PUF, Paris. 